\title{
Chaotic synchronization of vibrations of a coupled mechanical system consisting of a plate and beams
}

\begin{abstract}
In this paper mathematical model of a mechanical system consisting of a plate and either one or two beams is derived. Obtained PDEs are reduced to ODEs, and then studied mainly using the fast Fourier and wavelet transforms. A few examples of the chaotic synchronizations are illustrated and discussed.
\end{abstract}

\section{Keywords}

chaos, synchronization, plate, beam, wavelets
J. Awrejcewicz, A.V. Krysko, T.V. Yakovleva,

D.S. Zelenchuk, V.A. Krysko

J. Awrejcewicz

Department of Automation and Biomechanics, Technical University of Łódź, POLAND, 90-924 Lodz, 1/15 Stefanowski St.; awrejcew@p.lodz.pl

A.V. Krysko, T.V. Yakovleva, D.S. Zelenchuk

Engels Institute of Technology (Branch) Saratov State Technical University, Department of Higher Mathematics and Mechanics, RUSSIAN FEDERATION, 413100 Engels, Saratov region, Ploschad Svobodi 17, anton.krysko@gmail.com

V.A. Krysko

Saratov State Technical University, Department of Mathematics and Modeling, RUSSIAN FEDERATION, 410054 Saratov, Politehnicheskaya 77; tak@san.ru

\section{INTRODUCTION}

Nowadays a progress in investigation of chaotic dynamics in various branches of science like mechanics, physics, biology, chemistry, medicine, economy, etc. is achieved. In the field of mechanical continuous systems like beams/plates/shells there exists already a vast number of paper devoted to investigation of their bifurcational and chaotic behaviour. For instance, axially accelerating beams have been analysed in [1-4] using analytical approaches, whereas squared plates parametrically excited have been studied in [5-7] with respect to local and global bifurcations, the existence of heteroclinic and Shilnikov-type homoclinic orbits, Smale horseshoes and chaotic dynamics. Recently, in three companion papers chaotic dynamics of flexible plate and cylinder like panels of infinite length, rectangular spherical and cylindrical shells, closed cylindrical shells, axially symmetric plates, as well as spherical and conical shells have been studied (see [8-10]). A role of counter examples to deterministic behaviour of continuous systems plays the synchronization of non-linear dynamical processes.

The governing PDEs have been reduced to ODEs via the Finite Difference Method, the BubnovGalerkin Method and the Ritz Method. A few novel scenarios of transitions from regular to chaotic dynamics, as well as phase transitions chaos-hyper chaos and chaos-hyper chaos- hyper-hyper chaos have been reported, illustrated and discussed. Beginning from seminal works of Blekhman [11, 12], 
the today's development of synchronization goes beyond regular attractors (periodicity and quasiperiodicity) including synchronization of chaotic processes met in engineering objects.

One of important tasks of control of chaotic dynamics is devoted either to increase or decrease of a chaotization level of a being studied dynamical system. If one studies a dynamical system consisting of subsystems it is necessary to analyze chaotic synchronization of its parts (subsystems). For instance, a classical problem of control of cosmic construction relies on control of chaotic dynamics of its subsystems, and then step by step chaotic synchronization between elements (subsystems) is introduced, yielding finally the global synchronization of the cosmic object (a rocket or airplane). This work matches the so far briefly described non-linear phenomena, i.e. chaos and synchronization exhibited by continuous systems (here a plate and beam). Another important goal of this paper is that devoted to a study of non-linear interactions of structural members like beams and plates. Namely, we are aimed on a study of chaotic synchronization of multi-layered mechanical systems represented by beams and plates and coupled by boundary conditions. In this case on each time step a contact problem should be solved. Although chaotic dynamics of mechanical structures has been widely investigated (see monographs [13-15]), but the problem studied by us has not been investigated so far. It should be emphasized that during a classical investigation of chaotic dynamics mainly two characteristics have been applied, i.e. Fast Fourier Transform (FFT) and the Lyapunov exponents. However, as it has been pointed out in reference [16], FFT does not allow getting reliable results of transition from regular to chaotic dynamics. On the other hand the wavelet analysis allows separating of time evolutions of the frequency characteristics of vibrations. In this work we study a mechanical system consisting of two subsystems, i.e. a plate and two or one beam. We are aimed on analysis of chaotic synchronization and control of vibrations with a help of the wavelet approach. It should be noted that our study includes only one type of the boundary conditions. In our future studies it would be interesting also to include analysis of the influence of geometrical and physical nonlinearities on the obtained results. This problem has been partially studied in reference [19]. In addition, it would be also interesting to study behavior of structures taking into account other hypotheses. It should be noted, however, that we have studied already non-linear dynamics of beams using the Timoshenko hypothesis and wavelets in [20].

\section{MATHEMATICAL MODEL}

Mathematical model of chaotic vibrations of design-nonlinear two layered packet consisting of a plate and beams and being coupled via boundary conditions with loading of the form of $q=q_{0} \sin \left(\omega_{p} t\right)$ is studied (see Figures 1,2).

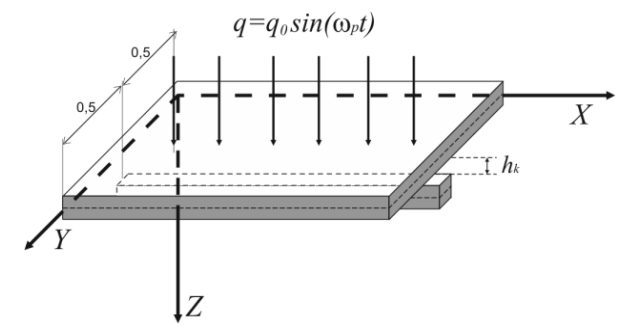

Figure 1 Two layered system consisting of a plate and a beam 


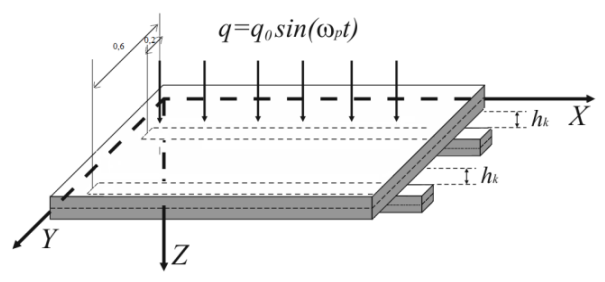

Figure 2 Two layered object consisting of a plate and two beams

Plate dynamics is governed by equation of the Lagrange type, whereas the beam is described by the Euler-Bernoulli equation, and the contact interaction obeys the Winkler model assumptions. The being studied construction is governed by the following non-dimensional equations

$$
\begin{gathered}
\frac{1}{12\left(1-\mu^{2}\right)}\left(\frac{1}{\lambda^{2}} \frac{\partial^{4} w_{1}}{\partial x^{4}}+\lambda^{2} \frac{\partial^{4} w_{1}}{\partial y^{4}}+2 \frac{\partial^{4} w_{1}}{\partial x^{2} \partial y^{2}}\right)+\frac{\partial^{2} w_{1}}{\partial t^{2}}+ \\
+\varepsilon \frac{\partial w_{1}}{\partial t}-q(t)-K\left(w_{1}-w_{2}-h_{k}\right) \Psi_{1}-K\left(w_{1}-w_{3}-h_{k}\right) \Psi_{2}=0 \\
\frac{1}{12} \frac{\partial^{4} w_{2}}{\partial x^{4}}+\frac{\partial^{2} w_{2}}{\partial t^{2}}+\varepsilon \frac{\partial w_{2}}{\partial t}+K\left(w_{1}-w_{2}-h_{k}\right) \Psi_{1}=0, \\
\frac{1}{12} \frac{\partial^{4} w_{3}}{\partial x^{4}}+\frac{\partial^{2} w_{3}}{\partial t^{2}}+\varepsilon \frac{\partial w_{3}}{\partial t}+K\left(w_{1}-w_{3}-h_{k}\right) \Psi_{2}=0
\end{gathered}
$$

where $\psi_{1}=\frac{1}{2}\left[1+\operatorname{sign}\left(w_{1}-h_{k}-w_{2}\right)\right]$ and $\psi_{2}=\frac{1}{2}\left[1+\operatorname{sign}\left(w_{1}-h_{k}-w_{3}\right)\right] ;$

$\mathrm{Y}_{1}=1$, if $\mathrm{w}_{1}>w_{2}+h_{k}$ is the contact between the plate and first beam, otherwise $\mathrm{Y}_{1}=0$; $\mathrm{Y}_{2}=1$, if $\mathrm{w}_{1}>w_{3}+h_{k}$ is the contact between the plate and a second beam; and finally $\mathrm{Y}_{2}=0$; $w_{1}, w_{2}, w_{3}$ are functions describing deflections of plate and beams, respectively; $K$ is the stiffness coefficient defined via a condition of non-penetration of the layers; $h_{k}$ is a gap between plate and beams; $q_{0}$ and $w_{p}$ is the amplitude and frequency of the harmonic excitation, respectively. It is assumed that both plate and beam materials are isotropic. System of equations (1) is reduced to the non-dimensional form through the following relations:

$$
x=\overline{a x}, y=a \bar{y} ; q=\bar{q} \frac{E(2 h)^{4}}{a^{2} b^{2}}, \tau=\frac{a b}{2 h} \sqrt{\frac{\rho}{E g}}, \lambda=\frac{a}{b},
$$

where $a, b$ denote plate dimensions regarding $x$ and $y$, respectively; $t$ denotes time, $e$ is the damping coefficient, $2 h$ denotes plate thickness, $m=0.3$ is Poisson's coefficient, $g$ denotes the Earth acceleration, $E$ is the Young's modulus, $q(x, y, t)$ denotes the transversal load. Bars over nondimensional parameters are omitted in equation (1). In addition, simple boundary conditions (2) as well as initial conditions (3) are attached of the form 


$$
\begin{gathered}
w_{m}=0 ;\left.\quad w_{m}^{\prime \prime}\right|_{x}=0 ; \text { for } x=0 ; 1 ; w_{m}=0 ;\left.\quad w_{1}^{\prime \prime}\right|_{y}=0 ; \text { for } y=0 ; 1 ; \\
\left.w_{1}(x, y)\right|_{t=0}=0,\left.\quad w_{2}(x)\right|_{t=0}=0,\left.\quad w_{3}(x)\right|_{t=0}=0,\left.\quad \dot{w}_{m}\right|_{t}=0, \quad m=1,2,3,
\end{gathered}
$$

where $m=1,2,3$ is the index corresponding to the plate and beams. Obtained system of nonlinear PDEs (1) is reduced to second order ODEs (1-3) through the Bubnov-Galerkin higher approximation order method. Deflections $w_{1}, w_{2}$ and $w_{3}$, being solutions to (1), are approximated by the following functions satisfying boundary conditions (2)

$$
\begin{aligned}
& w_{1}=\sum_{i=1}^{N} \sum_{j=1}^{N} A_{i j}^{1}(t) \sin (i \pi x) \sin (j \pi y), \\
& w_{2}=\sum_{i=1}^{N} A_{i}^{2}(t) \sin (i \pi x), \quad w_{3}=\sum_{i=1}^{N} A_{i}^{3}(t) \sin (i \pi x) .
\end{aligned}
$$

System of ODEs is solved via the fourth order Runge-Kutta method in time, and each layer is studied via standard techniques as time histories, phase portraits, Poincaré maps, fast Fourier and wavelet transforms as well as the Lyapunov exponents.

\section{ANALYSIS}

In what follows we apply the wavelet approach for detection of a synchronization regime of the considered mechanical dynamical system. In order to study a phase synchronization a notion of the chaotic synchronization phase is introduced [17-18]. Phase synchronization means that the phase of chaotic signals is synchronized, whereas amplitudes of signals are independent on each other and exhibit chaotization. Phase synchronization yields the frequencies synchronization, and hence the frequency of a chaotic signal is treated as the averaged velocity of phase changes. In the case of wavelet analysis the wavelet surface $W\left(s, t_{0}\right)=\left\|W\left(s, t_{0}\right)\right\| \exp \left[j \phi_{s}\left(t_{0}\right)\right]$ characterizes system dynamics on each time interval scale $s$ in a given time instant $t_{0}$. Magnitude $\left\|W\left(s, t_{0}\right)\right\|$ characterizes the occurrence and intensity of the corresponding time scale $s$ for a given time instant $t_{0}$. In addition, an integral energy distribution of the wavelet spectrum regarding time is introduced, i.e. $E(s)=\int\left\|W\left(s, t_{0}\right)\right\|^{2} d t_{0}$. Phase is defined as $\phi_{s}\left(t_{0}\right)=\arg W(s, t)$ for each of time scale $s$, which means that each time scale can be quantified through the associated with it the phase $\phi_{s}(t)$. On the other hand the phase synchronization yields synchronization on time scales $s$ as follows $\left\|f_{s_{1}}(t)-f_{s_{2}}(t)\right\|<$ const. In what follows we investigate the influence of the excitation amplitude as well as the beam versus plate position on the vibrational regime of our mechanical object. The

Latin American Journal of Solids and Structures 10(2013) $163-174$ 
following examples are studied: (i) beam is situated in the plate surface $(y=0.5)$ - see Figure 1 ; (ii) beam is shifted into plate edge with respect to the plate centre $(y=0.33)$; (iii) two parallel beams are symmetrically located versus plate centre $(y=0.4$ and $y=0.6)$; (iv) two parallel beams are non-symmetrically located versus plate centre $(y=0.2$ and $y=0.6)$ - see Figure 2 .

We study dynamical behavior of the two-layered mechanical system consisting of the plate and beams subjected to action of the load $q=q_{0} \sin \left(\omega_{p} t\right)$ and for the following fixed parameters: $h_{k}=0.01, e=1, w_{p}=5, l=1, K=5000$. In the earlier defined exemplary problems one may observe the phase synchronization of subsystems being in the chaotic regime. In Tables 1-6 dark color corresponds to synchronized frequencies, and they include the following characteristics: $a-$ time histories (signals); $b$ - phase portraits; $c-$ FFT power spectra; $d-2 \mathrm{D}$ Morlet wavelet-spectra; $f-3 \mathrm{D}$ Morlet wavelet-spectra of the plate and beam, respectively; $g$ - graphs of phase difference; $h$ - graphs of common plate, as well as first and second beams vibrations, respectively.

In first two cases for $q_{0}=0.065$ both plate and beam are in a contact, and although the plate exhibits harmonic vibrations with the frequency $\omega_{p}=5$, beam vibrations are damped. It means that in this case only the plate vibrates periodically. Increasing the excitation amplitude up to $q_{0}=0.07$ one observes a period three synchronization of plate and beam vibrations (Table1: $c_{1}$, $\left.c_{2}, g\right)$; In other words the reported in Table 1 the frequency power spectrum and wavelet frequency time evolution exhibit sub-harmonic vibrations of both plate and beam. Further increase of $q_{0}$, i.e. for,$q_{0}=0.08$ after a period three bifurcation the mechanical system transits to the chaotic state. It should be noted that for $q_{0}=0.1$ the chaotic orbit is exhibited and the associated FFT spectrum is broad band, whereas the beam vibrates with $w_{p}=5$, for $q_{0}=0.2$ a loop in the phase portrait is visible (Table $2: b_{1}$ ). Chaotic vibrations of plate and beam are associated with the dominating frequency $w_{p}=5$, which is indicated by the Fourier power spectra (Table 2: $c_{1}$, $c_{2}$ ) as well as 2D and $3 \mathrm{D}$ wavelet spectra (Table 2: $d_{1}, d_{2}, e_{1}, e_{2}$ ).

Let us study two-layered system in the case 3 , where beams are located on the same distance with respect to the plate centre. For $q_{0}=0.065$ and in result of initial contact of the plate with beams all three subsystems vibrate in a periodic manner. Increasing the excitation amplitude up to $q_{0}=0.07$, one may observe the system transition into chaos through period three bifurcations (Table 5: $c_{1}, c_{2}, c_{3}, f_{1}, f_{2}, f_{3}$ ). Beams vibrate in a synchronous manner under same loadings action (Table 5: $g$ ). For $q_{0}=0.2$ the chaotic orbit is shown in the phase plate portrait (Table 6: $b_{1}$ ), broad band parts of frequency power spectra are visible (Table 6: $c_{1}, c_{2}, c_{3}$ ), however phase portraits of two beams are different (Table $6: b_{2}, b_{3}$ ). In other words for $q_{0}=0.2$ vibration forms of two beams are non-symmetric one. 


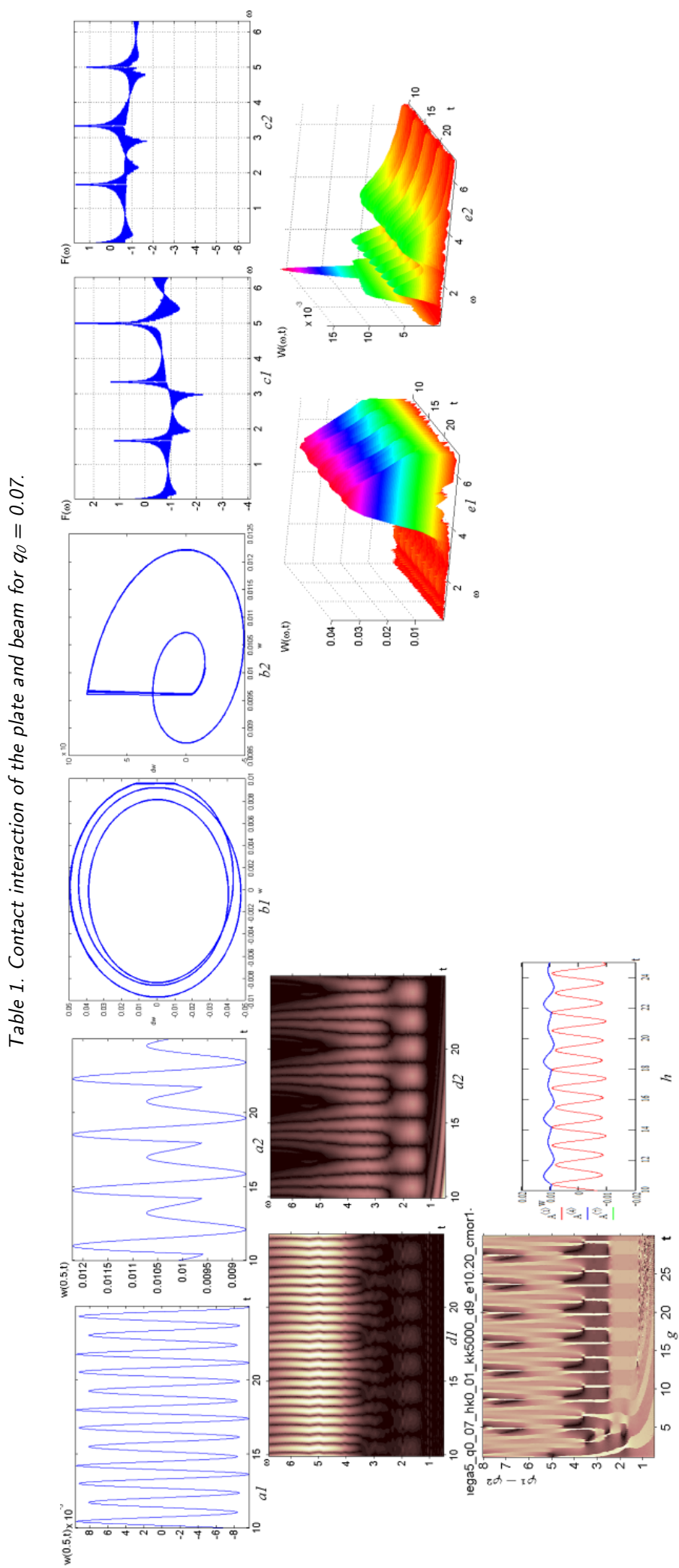

Latin American Journal of Solids and Structures 10(2013) $163-174$ 


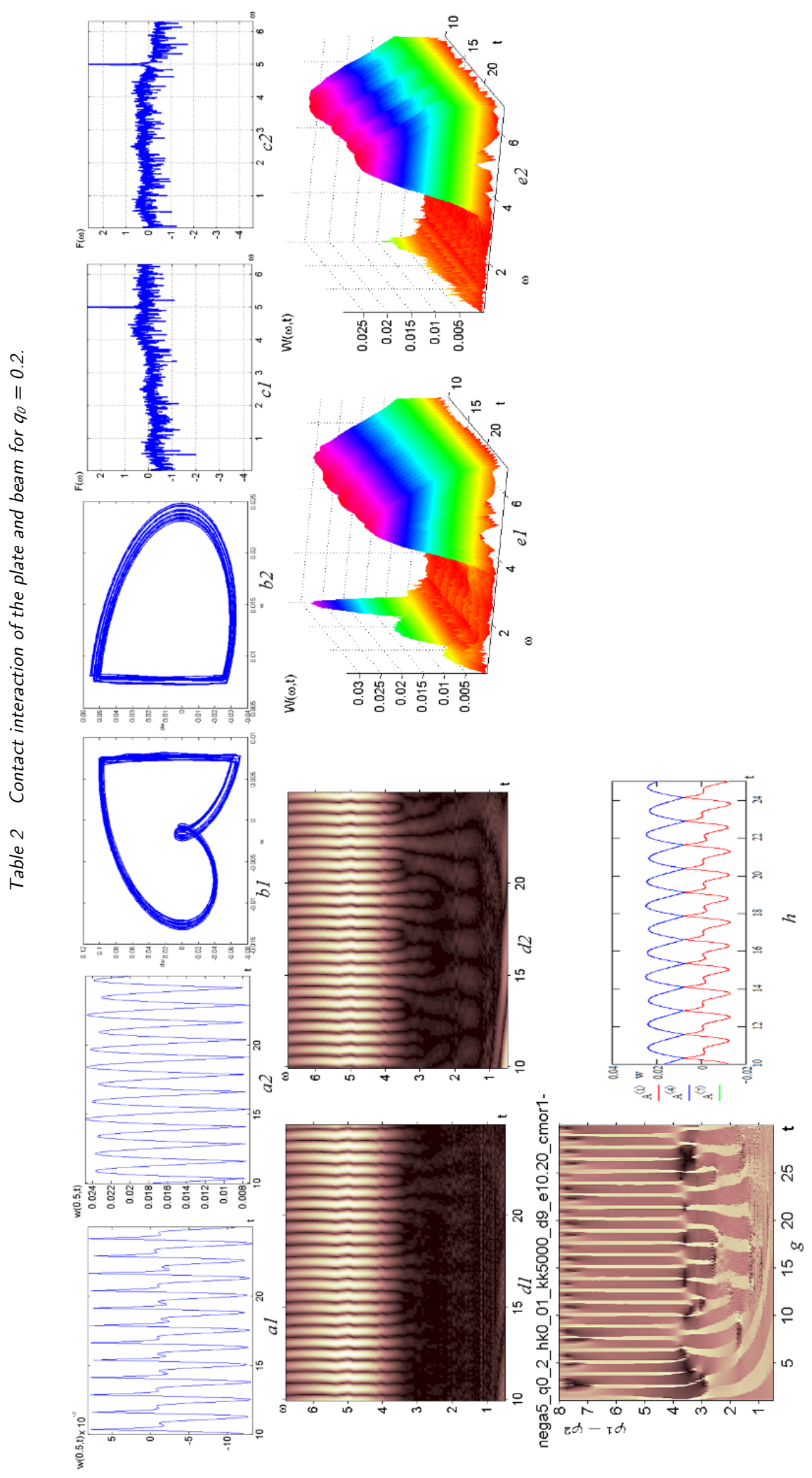

Latin American Journal of Solids and Structures 10(2013) $163-174$ 


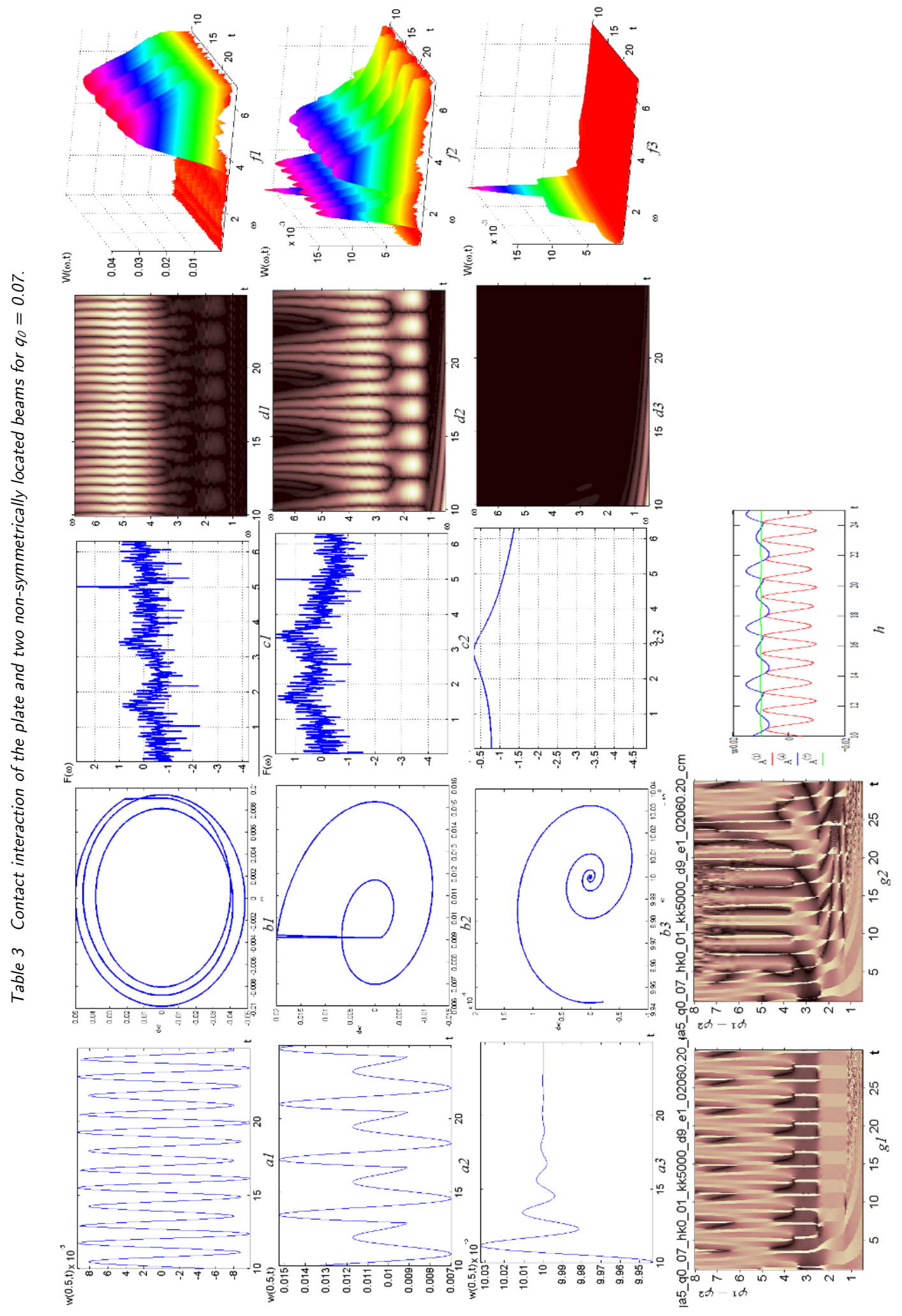

Latin American Journal of Solids and Structures 10(2013) $163-174$ 


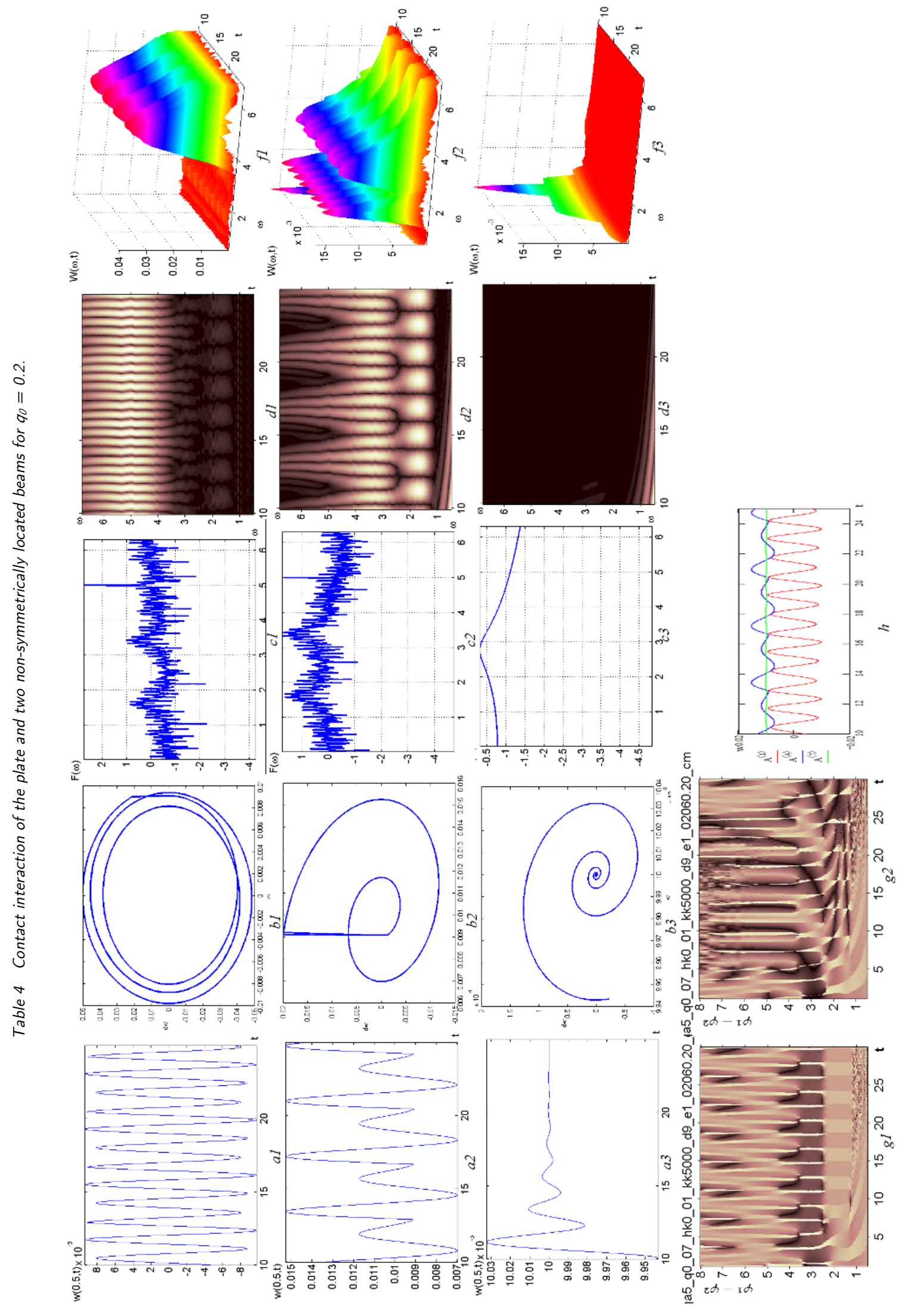

Latin American Journal of Solids and Structures 10(2013) $163-174$ 


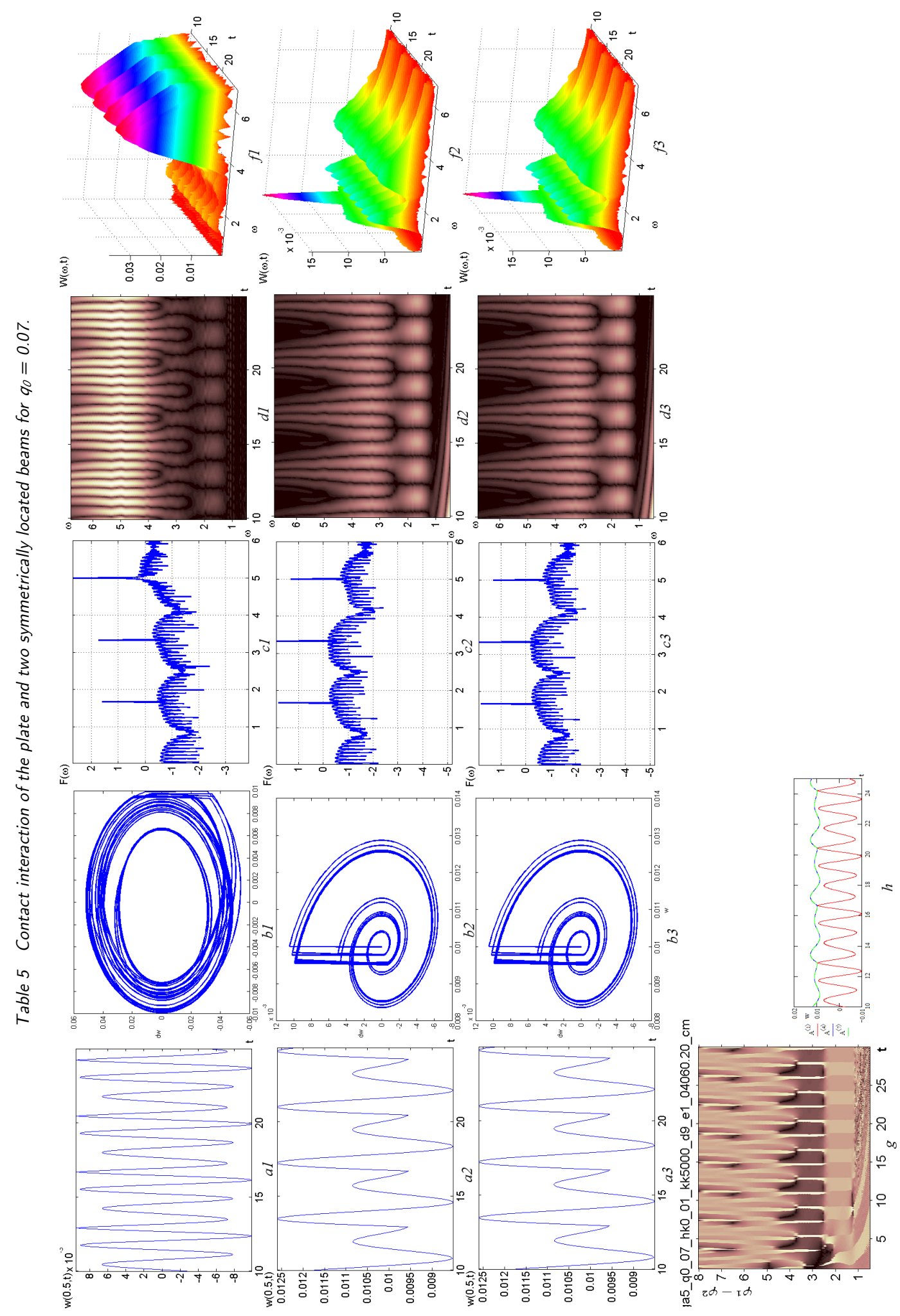

Latin American Journal of Solids and Structures 10(2013) $163-174$ 

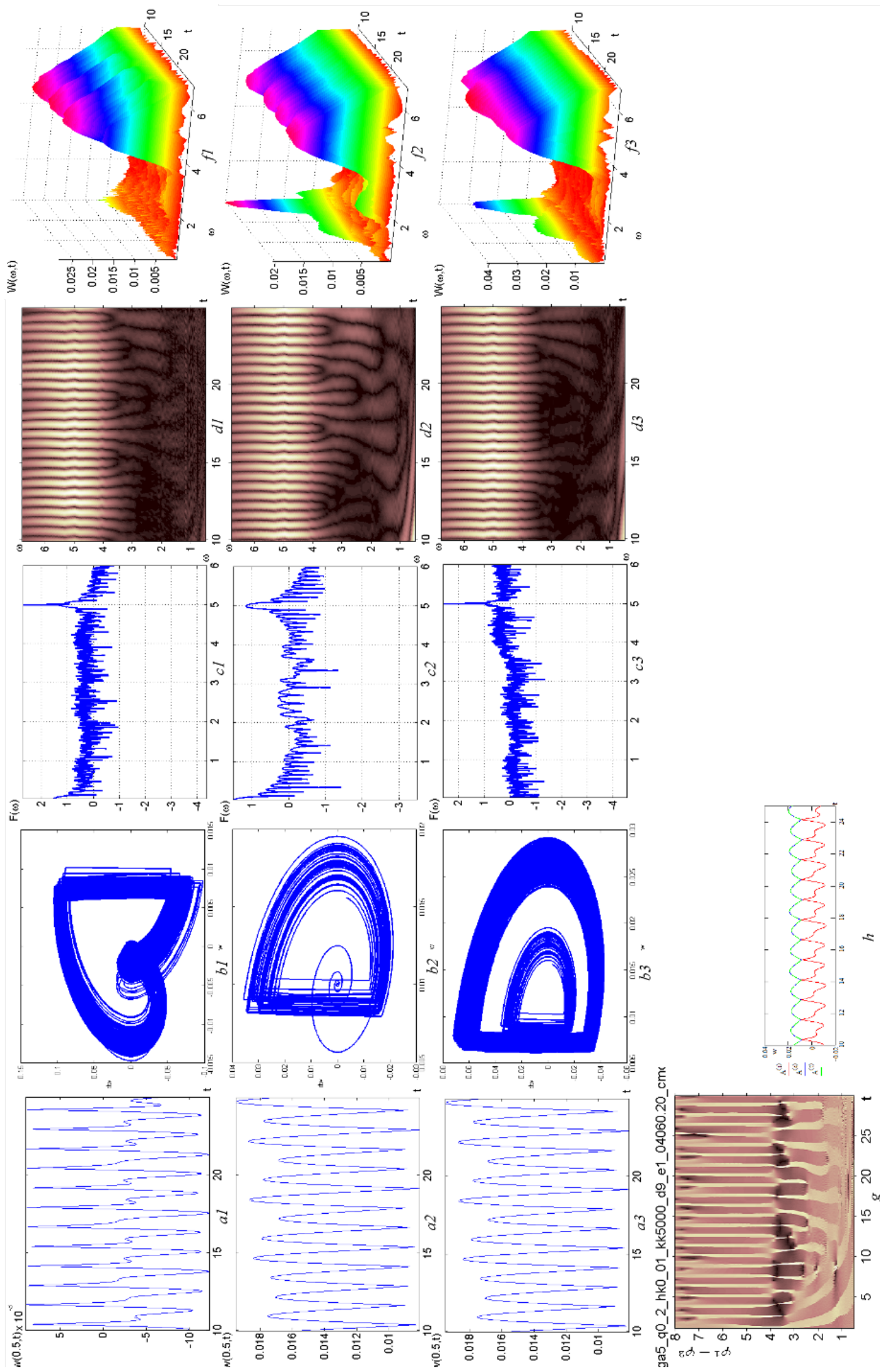

Latin American Journal of Solids and Structures 10(2013) 163 - 174 


\section{Concluding Remarks}

We have applied both classical tools of nonlinear dynamical systems (time and phase histories) as well as FFT and wavelets transforms to study interaction of a plate and either one or two beams.

First the governing equations are given, and then the problem has been reduced to study of ODEs solved via the $4^{\text {th }}$ order Runge-Kutta method. Examples of various bifurcational and chaotic dynamics of the studied objects putting emphasis on the chaotic synchronization are reported in section 2 .

Acknowledgements The paper was presented at the 11th Conference on Dynamical Systems - Theory and Applications, December 5-8, 2011 in Łódź, Poland. The support of the Alexander von Humboldt Award is acknowledged by J. Awrejcewicz.

\section{References}

[1] Revindra B., Zhu WD., Low dimensional chaotic response of axially accelerating continuum in the supercritical regime. Arch. Appl. Mech. 1998;68:195-205.

[2] Pellicano F., Vestroni F., Complex dynamics of high-speed axially moving systems. J.Sound Vib. 2002;258: 31-44.

[3] Chen LQ., Wu J., Zu JW., The chaotic response of the viscoelastic travelling string: an integral constitutive law. Chaos, Solitons and Fractals 2004;21: 359-57.

[4] Yang XD., Chen LQ., Bifurcation and chaos of an axially accelerating viscoelastic beam. Chaos, Solitons and Fractals 2005;33:249-58.

[5] Yang XL., Sethna PR., Local and global bifurcations in parametrically excited vibrations of nearly square plates. Int. J. Non-linear Mech. 1991;26:199-220.

[6] Feng ZC., Sethna PR., Global bifurcations in the motion of parametrically excited thin plate. Nonlinear Dyn. 1993;4:389-408.

[7] Yeh YL., Chen CK., Lai HY., Chaotic and bifurcation dynamics of a simply supported thermo-elastic circular plate with variable thickness in large deflection. Chaos, Solitons and Fractals 2003;15:811-29.

[8] Awrejcewicz J., Krysko V.A., Papkova I.V., Krysko A.V., Routes to chaos in continuous mechanical systems. Part 1. Mathematical models and solution methods. Chaos, Solitons and Fractals 2012 (to appear).

[9] Awrejcewicz J., Krysko V.A., Papkova I.V., Krysko A.V., Routes to chaos in continuous mechanical systems. Part 2. Modelling transitions from regular to chaotic dynamics. Chaos, Solitons and Fractals 2012 (to appear).

[10] Awrejcewicz J., Krysko V.A., Papkova I.V., Krysko A.V., Routes to chaos in continuous mechanical systems. Part 3. The Lyapunov exponent, hyper, hyper-hyper and spatial-temporal chaos. Chaos, Solitons and Fractals 2012 (to appear).

[11] Blekhman I.I, Synchronization of Dynamical Systems. Nauka, Moscow 1971, in Russian.

[12] Blekhman I.I, Synchronization in the Nature and Technics. Nauka, Moscow 1981, in Russian.

[13] Awrejcewicz J., Krysko V.A., Chaos in Structural Mechanics. Springer, Berlin, London, 2008.

[14] Awrejcewicz J., Krysko V.A., Krysko A.V., Thermodynamics of Plates and Shells. Springer, Berlin, London, 2007.

[15] Awrejcewicz J.A., Krysko V.A., Vakakis A.F., Nonlinear Dynamics of Continuous Elastic System. Springer, Berlin, 2004.

[16] Awrejcewicz J., Krysko V.A., Soldatov V., Krysko A.V., Analysis of the nonlinear dynamics of the Timoshenko flexible beams using wavelets. J. Comp. and Nonlin. Dyn. (to appear).

[17] Osipov G.V., Pikovsky A.S., Rosenblum M.G., Kurths J., Phase synchronization effect in a lattice of non-identical Rössler oscillators. Physical Review E. 1997;55:2253-2361.

[18] Pikovsky A.S., Rosenblum M.G., Kurths J., Synchronization: a Universal Concept in Nonlinear Sciences. Cambridge University Press 2001.

[19] J. Awrejcewicz, A. V. Krysko, V. V. Bochkarev, T. V. Babenkova, I. V. Papkova, J. Mrozowski, Chaotic vibrations of two-layered beams and plates with geometric, physical and design non-linearities. International Journal of Bifurcation and Chaos, 2011, 21, 2837-2851.

[20] J. Awrejcewicz, A. V. Krysko. V. V. Soldatov, V. A. Krysko, Analysis of the nonlinear dynamics of Timoshenko flexible beams using wavelets. Journal of Computational and Nonlinear Dynamics, 2012, 7, 1-14.

Latin American Journal of Solids and Structures 10(2013) $163-174$ 